\title{
Quimioterapia adjuvante em câncer de endométrio precoce: Revisão sistemática e metanálise
}

\author{
Adjuvant chemotherapy in early endometrial cancer: Systematic review and meta-analysis \\ Quimioterapia adyuvante en cáncer de endometrio precoz: Revisión sistemática y \\ metanálisis
}

Ricardo Baer Filho1*, Rubens Moura Campos Zeron¹, Bruna Regina Oliveira Martins .

\begin{abstract}
RESUMO
Objetivo: Explorar se o tratamento quimioterápico adjuvante, em pacientes com câncer de endométrio operados em estadios iniciais, traz benefícios. Métodos: Identificação dos estudos pelas bases de dados eletrônicas: MEDLINE, EMBASE, COCHRANE e LILACS. Resultados: Analisou-se um total de 560 pacientes sendo que destas, 168 foram submetidas a quimioterapia, 84 submetidas a quimioterapia e radioterapia e 308 do grupo comparação sendo 72 com radioterapia e 236 não receberam nenhum tratamento adjuvante. Não houve diferença estatisticamente significativa entre o uso de quimioterapia adjuvante em câncer de endométrio em estadios iniciais e a sobrevivência global em cinco anos (RD=0.19 com IC 95\%, -0.39 a 0.02). $\mathrm{Na}$ composição da metanálise, em 3 estudos foi demonstrado maior sobrevivência global em 5 anos com o uso de quimioterapia quando comparado ao grupo controle sem quimioterapia e em 2 estudos não houve diferença entre grupos. Em relação a recorrência, não foi significativo o uso de quimioterapia adjuvante ( $R D=0.4$ com IC 95\%, - 0.33 a 0.06). Conclusão: Não foi possível definir benefício ou malefício com a quimioterapia adjuvante em câncer de endométrio precoce em relação a sobrevivência global e recorrência.
\end{abstract}

Palavras-chaves: Neoplasias do Endométrio, Quimioterapia Adjuvante, Análise de Sobrevida.

\begin{abstract}
Objective: To investigate whether adjuvant chemotherapeutic treatment in patients with operated endometrial cancer in early stages has benefits. Methods: Identification of the studies in electronic databases: MEDLINE, EMBASE, COCHRANE and LILACS. Results: A total of 560 patients were analyzed. Of these, 168 were submitted to chemotherapy, 84 underwent chemotherapy and radiotherapy and 308 of the comparison group, being 72 with radiotherapy and 236 receiving no adjuvant treatment. There was no statistically significant difference between the use of adjuvant chemotherapy for endometrial cancer in early stages and overall survival in five years (RD $=0.19$ with $95 \% \mathrm{Cl},-0.39$ to 0.02 ). In the meta-analysis, 3 studies showed greater overall survival in five years with the use of chemotherapy when compared to the control group without chemotherapy and in 2 studies there was no difference between groups. Regarding recurrence, the use of adjuvant chemotherapy ( $\mathrm{RD}=0.14$ with $95 \% \mathrm{Cl},-0.33$ to 0.06 ) was not significant. Conclusion: It was not possible to define benefit or harm with adjuvant chemotherapy in early endometrial cancer in relation to global survival and recurrence.
\end{abstract}

Key words: Endometrial Neoplasms, Adjuvant Chemotherapy, Survival Analysis.

\section{RESUMEN}

Objetivo: Investigar si el tratamiento quimioterápico adyuvante, en pacientes con cáncer de endometrio operados en estadios iniciales, trae beneficios. Métodos: Identificación de los estudios en las bases de datos electrónicas: MEDLINE, EMBASE, COCHRANE y LILACS. Resultados: Se analizó un total de 560 pacientes que de ellos, 168 fueron sometidos a quimioterapia, 84 sometidos a quimioterapia y radioterapia y 308 del grupo comparado siendo 72 con radioterapia y 236 no recibieron ningún tratamiento adyuvante. No hubo diferencia estadísticamente significativa entre el uso de quimioterapia adyuvante en cáncer de endometrio en

${ }^{1}$ Centro Universitário Lusíada, Santos-SP. *E-mail: baerfilho@gmail.com

SUBMETIDO EM: 5/2019

ACEITO EM: 6/2019

PUBLICADO EM: 7/2019

REAS | Vol. 11(13) | e1002 | DOI: https://doi.org/10.25248/reas.e1002.2019 Página 1 de 9 
estadios iniciales y la supervivencia global en cinco años ( $R D=0,19$ con IC95\%, $-0,39$ a 0,02). En la composición del metanálisis, en 3 estudios se demostró mayor supervivencia global en cinco años con el uso de quimioterapia cuando comparado al grupo control sin quimioterapia y en 2 estudios no hubo diferencia entre grupos. En relación a la recurrencia, no fue significativo el uso de quimioterapia adyuvante $(\mathrm{RD}=0,14$ con IC95\%, -0,33 a 0,06). Conclusión: No fue posible definir beneficio o maleficio con la quimioterapia adyuvante en cáncer de endometrio precoz en relación a la supervivencia global y recurrencia.

Palabras clave: Neoplasias del Endometrio, Quimioterapia Adyuvante, Análisis de Sobrevida.

\section{INTRODUÇÃO}

O carcinoma de endométrio é o câncer ginecológico mais comum do mundo desenvolvido. A maioria das pacientes se encontra na pós-menopausa, com apenas $25 \%$ de pré-menopausadas e $3 \%$ de pacientes abaixo dos 40 anos de idade. Os fatores de risco para o desenvolvimento do câncer de endométrio incluem: obesidade, nuliparidade, menopausa tardia, diabetes mellitus, estrogenioterapia exclusiva, terapia com tamoxifeno e uso de contraceptivos orais. Os tumores epiteliais compreendem $97 \%$ dos casos, e o adenocarcinoma endometrioide é o tipo histológico mais comum (90\%). Os outros $10 \%$ compreendem o carcinoma de células claras e o carcinoma seroso papilífero (BOER SM, et al., 2016).

A maioria das mulheres com câncer de endométrio apresenta-se no estádio I (de acordo com o estadiamento proposto pela Federação Internacional de Ginecologistas e Obstetras, FIGO) e tem bom prognóstico, com uma taxa de sobrevida global superior a $90 \%$. Com exceção das mulheres com doença localmente avançada ou metastática, o tratamento definitivo para o carcinoma endometrial é a histerectomia total abdominal e salpingo-ooforectomia bilateral seguida ou não de linfadenectomia pélvica e para-aórtica. A decisão pela utilização de tratamento adjuvante depende de alguns fatores de risco (BRINTON LA, et al., 2013).

Com o objetivo de impedir ou diminuir a taxa de aparecimento de recidivas, propõe-se a realização de terapêuticas adjuvantes. Dentre elas, a radioterapia pélvica, a braquiterapia vaginal e a quimioterapia têm sido utilizadas como tratamentos complementares à cirurgia. Porém, a relevância dessas terapias permanece controversa, particularmente na doença em estádio I, dada a excelente taxa de sobrevida global dessas pacientes. Outro aspecto a ser considerado é que não existem muitos estudos clínicos randomizados publicados que avaliem exclusivamente o papel da quimioterapia no tratamento do câncer endometrial inicial (VALEJO FAM e TIEZZI DG, 2009).

O uso de tratamento adjuvante sistêmico em câncer de endométrio é uma decisão individualizada com base na avaliação de fatores de risco que aumentam o potencial de recidiva locorregional e/ou metástase à distância, como: estadio clínico (FIGO), idade, características histológicas, grau de diferenciação, profundidade, extensão e invasão do espaço linfovascular (CREASMAN WT, et al., 2004; CREUTZBERG CL, et al., 2004; HIRAI M, et al., 2002). Deve-se considerar o equilíbrio entre os benefícios esperados e os riscos de toxicidade dos tratamentos adjuvantes. Estabelecer o benefício de um tratamento mais intensivo e o efeito em termos de morbidade e efeito agregados sobre a qualidade de vida relacionada à saúde são essenciais (BOER SM, et al., 2016).

Uma série de ensaios clínicos randomizados confirmaram que a radioterapia adjuvante pode reduzir o risco de recorrência loco-regional em pacientes com diagnóstico de doença inicial e de alto risco; no entanto, não foi suficiente para aumentar a sobrevivência global (KELLY MG, et al.,2005; KEYS HM, et al., 2004).

Alguns ensaios clínicos mostraram que a quimioterapia adjuvante melhorou significativamente a sobrevida global em pacientes com câncer de endométrio comparado com a radiação adjuvante, no entanto, a maioria desses estudos incluiu pacientes com doença avançada (KODAMA J, et al., 2007; KUOPPALA T, et al., 2008; LAN C, et al., 2015). Atualmente, dados limitados estão disponíveis sobre a eficácia de pacientes com quimioterapia adjuvante com câncer de endométrio em estágio inicial. O objetivo deste estudo foi explorar se o tratamento quimioterápico adjuvante em pacientes com câncer de endométrio operados em estadios iniciais traz benefícios.

REAS | Vol. 11(13) | e1002 | DOI: https://doi.org/10.25248/reas.e1002.2019 Página 2 de 9 


\section{MÉTODOS}

\section{Protocolo e registro}

Essa revisão sistemática e meta-análise foram realizadas de acordo com a recomendação PRISMA e foi registrada no protocolo de revisão PROSPERO (CRD42018083904).

\section{Fontes de informação e estratégia de busca}

Foram utilizadas estratégias de busca à literatura, descritas no Quadro 1, que incluiu pesquisas efetuadas nas bases MEDLINE, EMBASE, COCHRANE e LILACS. As buscas foram finalizadas em março de 2019.

Quadro 1 - Bases utilizadas e estratégia de busca.

MEDLINE: (endometrial neoplasm OR endometrial carcinoma OR endometrial cancer OR endometrium cancer OR cancer of the endometrium OR carcinoma of endometrium) AND (adjuvant chemotherapy OR adjuvant drug therapy OR combined model therapy OR combined modality therapy OR Paclitaxel OR Carboplatin OR Doxorubicin OR Cisplatin)

EMBASE: (endometrial neoplasm OR endometrial carcinoma OR endometrial cancer OR endometrium cancer OR cancer of the endometrium OR carcinoma of endometrium)

COCHRANE: Endometrial Neoplasm AND Adjuvant

LILACS: Endometrial Neoplasm AND Adjuvant

Fonte: Realizada pelos autores, 2019.

\section{Critério de elegibilidade}

Foram incluídos estudos do tipo coorte retrospectivo e ensaio clínico randomizado. Não foi feita restrição quanto ao ano de publicação. Fez-se restrição quanto ao idioma, sendo considerados apenas artigos na língua inglesa. Estudos aos quais não havia acesso ao texto completo foram excluídos da análise. O principal critério de inclusão e exclusão foram os componentes do P.I.C.O (abreviação do inglês: Paciente, Intervenção, Controle e Desfecho). Estudos que não possuíam grupo comparação foram excluídos. Foi realizada a Revisão Sistemática que teve como objetivo avaliar se a quimioterapia adjuvante em câncer de endométrio em estadio I e II está relacionada ao aumento da sobrevida global ou redução de recorrência.

Tabela 1 - Escala de Newcastle-Ottawa para avaliação da qualidade de estudos Coorte.

\begin{tabular}{|c|c|c|c|c|}
\hline Critérios & 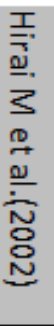 & 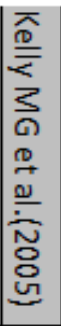 & 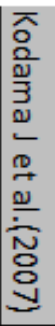 & 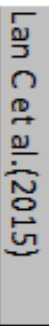 \\
\hline \multicolumn{5}{|l|}{ Seleção } \\
\hline Representatividade da amostra da coorte exposta & * & * & * & * \\
\hline \multicolumn{5}{|l|}{ Seleção da coorte não exposta } \\
\hline Determinação da exposição & * & * & * & * \\
\hline Demonstração de que o resultado de interesse não estava presente no ínicio do estudo. & * & * & * & * \\
\hline \multicolumn{5}{|l|}{ Comparabilidade } \\
\hline Comparação de coortes com base no desenho ou análise & & & & * \\
\hline \multicolumn{5}{|l|}{ Desfecho } \\
\hline Avaliação dos resultados & * & * & * & * \\
\hline Foi um acompanhamento longo o suficiente para que os desfechos ocorrecem & * & * & * & * \\
\hline Adequação do acompanhamento de coortes & * & * & * & * \\
\hline
\end{tabular}

Fonte: Critérios em http://www.ohri.ca/programs/clinical_epidemiology/oxford.asp. Classificação dos critérios realizada pelos autores, 2019. 


\section{Seleção dos estudos}

O processo de recuperação dos trabalhos na MEDLINE, LILACS e COCHRANE foi realizado por meio de dois subgrupos. Cada subgrupo foi composto por dois pesquisadores que atuaram de forma independente $e$ quando houve divergência entre pesquisadores nas etapas de: seleção por resumos e do texto na íntegra, 0 outro subgrupo foi consultado. Na fase de seleção por títulos os artigos divergentes foram selecionados.

\section{Risco de vieses e Avaliação da qualidade}

Para a análise de vieses nos estudos coorte foi aplicado o "NOS-Newcastle-Ottawa Scale" (Tabela 1) e para o ensaio clínico randomizado o "Cochrane Riskof Bias Tool for Randomized Controlled Trials" (Tabela 2).

Tabela 2 - Ferramenta Cochrane de Risco de Vieses para Ensaios Clínicos Randomizados.

\begin{tabular}{lc} 
Geração de Sequência Aleatória & $\begin{array}{c}\text { Kuoppala T et al. (2008) } \\
\text { Nível de Risco }\end{array}$ \\
$\begin{array}{l}\text { Viés de seleção (viés de alocação para intervenção) devido à geração inadequada da } \\
\text { sequência aleatória }\end{array}$ & Baixo \\
\hline Ocultação da Alocação &
\end{tabular}

Viés de seleção (viés de alocação para intervenção) devido à ocultação inadequada da alocação antes de designação

Baixo

Viés de Notificação

Viés de notificação devido a notificação do desfecho

Baixo

\section{Outros Vieses}

Viés devido a problema não oculto em outra parte da tabela

Baixo

\section{Cegamento de Participantes e Pessoal}

Viés de desempenho devido ao conhecimento das intervençőes alocadas pelos

participantes durante o estudo

Alto

\section{Cegamento da Avaliação dos Resultados}

Viés de detecção devido ao conhecimento das intervençoes alocadas dos desfechos pelos avaliadores

Baixo

\section{Dados de Desfecho Incompleto}

Viés de atrito devido à quantidade, natureza ou utilização de dados de desfecho incompleto

Baixo

Fonte: Critérios em https://methods.cochrane.org. Classificação dos critérios realizada pelos autores, 2019.

\section{Análise estatística}

Para realização da metanálise, foi empregado o RevMan 5.3. Para as estimativas dos cálculos, utilizou-se diferença de risco para variáveis dicotômicas com o teste de Cochran-Mantel-Haenszel e $95 \%$ de intervalo de confiança (IC95\%). A apresentação foi feita por Forest Plot. Foi considerado homogeneidade entre os estudos quando $\mathrm{I}^{2}<50$ e utilizou-se o modelo com efeitos fixos. A heterogeneidade foi considerada elevada quando $I^{2} \geq 50$ e, nesses casos, o modelo randômico foi utilizado. 


\section{RESULTADOS}

\section{Seleção dos estudos}

Realizou-se a seleção em março de 2019 e foram identificados 4312 artigos. Após a exclusão dos artigos duplicados, foi realizada a seleção por análise de títulos e resumo. Quatro mil duzentos e noventa e quatro artigos foram excluídos por não preencherem o critério de inclusão.

Tabela 3 - Artigos com textos completos excluídos e justificativas.

Artigo

Aoki Y et al. (2004)

Boer SM et al. (2016)

Burke TW et al. (1994)

Fehr MK et al. (1999)

Fehr MK, Strech MS (2006)

Miyahara D et al. (2016)

Mundt AJ et al. (2001)

Nakamura T et al. (2000)

Noda K et al. (1987)

Pustilnik T, Burke TW (2000)

Stringer CA et al. (1990)

Tsunoda $\mathrm{H}$ et al.(1996)

Yamada K et al. (2013)
Motivo de Exclusão

O grupo de comparação foi criado a partir da contraindicação por tratamento citotóxico $(n=8)$ e recusa de pacientes em submeter a quimioterapia $(n=5)$. A recomendação a quimioterapia foi individualizada e conforme achados patológicos

Avalia a qualidade de vida e toxidade após tratamento adjuvante

Não houve grupo de comparação. Todos receberam quimioterapia

Texto completo disponível apenas em Alemão

Texto completo disponível apenas em Alemão

Compara grupo de alto e médico risco usando quimioterapia

Não houve grupo de comparação. Todos receberam quimioterapia

Texto completo não disponível e artigo em Japonês

Texto completo não disponível e artigo em Japonês

Texto completo não disponível

Não houve grupo de comparação. Medição da atividade e toxidade da quimioterapia sistêmica

Texto completo não disponível e artigo em Japonês

Testou diferentes doses de paclitaxel e carboplatin

Fonte: Dados coletados pelos autores, 2019.

Figura 1 - Fluxograma para seleção dos estudos

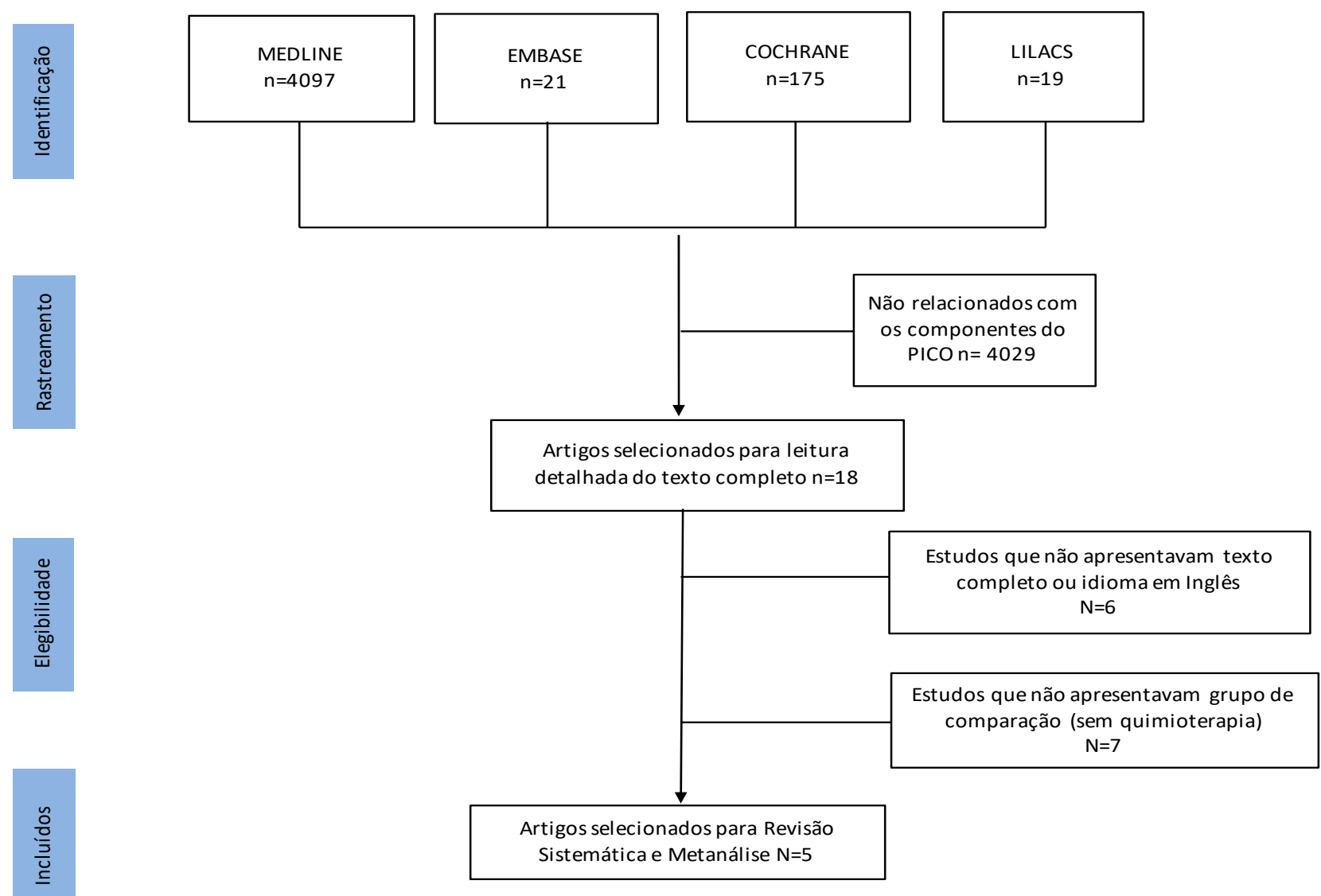

Fonte: Dados coletados pelos autores, 2019.

REAS | Vol. 11(13) | e1002 | DOI: https://doi.org/10.25248/reas.e1002.2019 Página 5 de 9 
Posteriormente, seguiu-se a análise detalhada de 18 artigos e foram excluídos 7 por não apresentarem grupo de comparação e 6 artigos foram excluídos por não possuírem texto completo em inglês (Tabela 3). Dessa forma, 5 estudos foram incluídos para revisão sistemática (Figura 1).

\section{Características dos estudos}

Quatro artigos do tipo Coorte Retrospectiva e um Ensaio Clínico Randomizado foram incluídos. Analisouse um total de 560 pacientes sendo que destas, 168 foram submetidas a quimioterapia, 84 submetidas a quimioterapia e radioterapia e 308 do grupo comparação sendo 72 com radioterapia e 236 não receberam nenhum tratamento adjuvante (Tabela 5).

Tabela 5 - Características dos estudos selecionados.

\begin{tabular}{ccccccccccc}
\multicolumn{1}{c}{ Artigo } & Tipo de Estudo & Tipo Histológico & Estadio & Grau do Tumor & Intervenção Comparação & N.I & N.C & $\begin{array}{c}\text { Tempo de } \\
\text { Acompanhamento }\end{array}$ \\
\hline Hirai M et al. (2002) & CR & END, SER, MUN, CLA & IA,IB,IC & $1-3$ & QMT & STA & 23 & 31 & 5 e 10 anos & SG, SLD,T \\
Kelly MG et al. (2005) & CR & SER & IA,IB,IC & & QMT & STA & 32 & 32 & 5 anos & SG,SLD \\
Kodama J et al. (2007) & CR & END & I,IIIIIIA & $1-3$ & QMT & STA & 58 & 109 & 5 anos & SG,SLD \\
Lan C et al. (2015) & CR & END & IB, II & $1-3$ & QMT & STA & 55 & 64 & 5 anos & SG,SLD \\
Kuoppala T et al. (2008) & ECR & CLA, ESC & IA+B, IC, IIA+B, IIIA & $1-3$ & QMT+RT & RT & 84 & 72 & 5 anos & SG,SLD,T \\
\hline
\end{tabular}

END = adenocarcioma endometrioide, $\mathrm{SER}=$ Adenocarcinoma Seroso-papilífero, $\mathrm{MUN}=$ Adenocarcinoma Mucinoso, $\mathrm{CLA}=$ Adenocarcionoma de célula clara, $\mathrm{ESC}=$ Carcinoma Escamoso, QMT = Quimioterapia, $\mathrm{RT}=$ Radioterapia, STA= Sem tratamento Adjuvante, $\mathrm{SG}=$ Sobrevida global, SLD= Sobrevida livre de doença, $\mathrm{T}=$ Toxidade, N.I= Número do grupo de intervenção, N.C= Número grupo controle.

Fonte: Dados coletados pelos autores, 2019.

\section{Síntese dos Resultados}

Não houve diferença estatisticamente significativa entre o uso de quimioterapia adjuvante em câncer de endométrio em estadios iniciais e a sobrevivência global em cinco anos ( $R D=0.19$ com IC95\%, -0.39 a 0.02). Em 3 estudos foi demonstrado maior sobrevivência global em 5 anos com o uso de quimioterapia quando comparado ao grupo controle sem quimioterapia e em 2 estudos não houve diferença entre grupos (Figura 2).

Figura 2 - Forest Plot: sobrevida global em 5 anos de pacientes submetidos à quimioterapia em relação aos não submetidos.

\begin{tabular}{lcccccccc} 
& \multicolumn{1}{c}{$\begin{array}{c}\text { Quimio Adjuv } \\
\text { Autor, Ano }\end{array}$} & $\begin{array}{c}\text { Controle } \\
\text { Eventos }\end{array}$ & Total & Eventos & Total & Peso & Intervalo de Confiança 95\%
\end{tabular}

Fonte: Dados coletados pelos autores, 2019. 
Em relação a recorrência não foi significativo o uso de quimioterapia adjuvante (RD=0.14 com IC95\%, 0.33 a 0.06). A diferença foi estatisticamente significativa em 2 estudos e em 3 estudos não houve diferença significativa (Figura 3).

Figura 3 - Florest Plot: Incidência de recorrência de câncer em 5 anos de pacientes submetidos a quimioterapia em relação aos não submetidos.

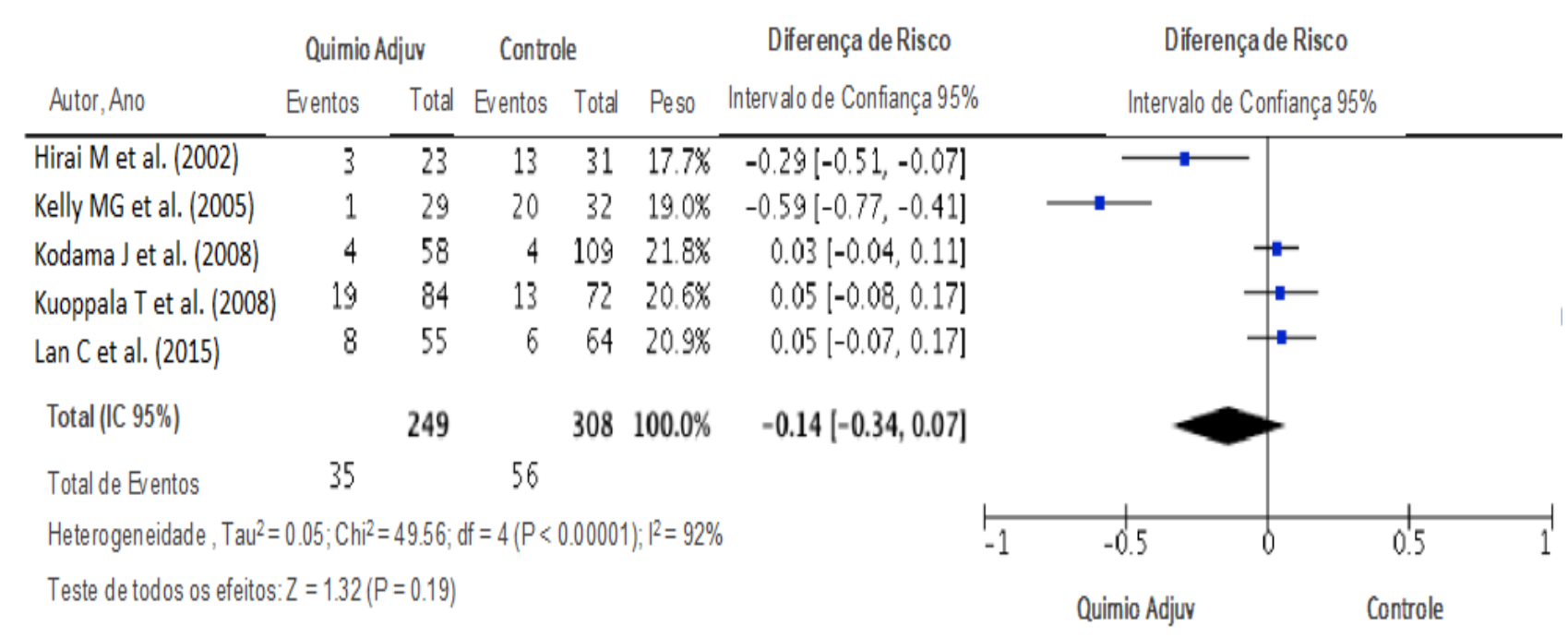

Fonte: Dados coletados pelos autores, 2019.

\section{DISCUSSÃO}

O câncer de endométrio geralmente tem um bom prognóstico porque a maioria dos casos é diagnosticada no estadio I, contudo, também é possível identificar subgrupos de pacientes com câncer de endométrio em estágio inicial com um prognóstico ruim. Não existem muitos estudos clínicos randomizados publicados que avaliem exclusivamente o papel da quimioterapia no tratamento do câncer endometrial inicial. O GOG-177 foi o primeiro estudo de fase III sobre quimioterapia em câncer de endométrio avançado ou recorrente que mostrou uma vantagem de sobrevivência (HOWARD DH, et al., 2009).

No presente estudo, não foi encontrada diferença no uso de quimioterapia adjuvante em câncer de endométrio em estadios iniciais e a sobrevivência global em cinco anos. Porém, quando observamos pacientes com carcinoma seroso papilar uterino em estadios IA, IB e IC, houve redução de risco em -0.53 (95\% IC, $-0.71 ;-0.36)$ o que pode estar relacionado com alta taxa de incidência de doença extrauterina e baixas taxas de sobrevida em cinco anos em comparação ao tipo endometrioide (KELLY MG, et al., 2005; MAGGI R, et al., 2006).

Hirai $\mathrm{M}$ et al.(2002) encontrou benefício no tratamento de quimioterapia adjuvante com relação a sobrevivência global em 5 anos quando comparado ao não tratamento em grupo de pacientes com invasão linfa-vascular (95\% IC, -0.51; -0.07). O grupo de pacientes possuia diferentes tipos histológicos sendo $85 \%$ Adenocarcinoma endometrio. O número de pacientes em que foi comparado o uso de quimioterapia adjuvante foi relativamente pequeno (grupo de intervenção $n=23$ e grupo controle $n=31$ ) e não houve critério de seleção dos grupos.

Em relação à recorrência da doença não houve diferença estatisticamente significativa quanto ao uso de quimioterapia adjuvante $(95 \%$ IC, $-0.34 ; 0.07)$. Em dois estudos que compuseram a metanálise, o uso de quimioterapia adjuvante demonstrou a redução na recorrência da doença. No tipo histológico papilífero seroso, apresentou redução de recorrência significativa (95\% IC, $-0.77 ;-0.41)$ sendo que este tipo histológico apresenta maiores taxas de recorrência (KELLY MG, et al., 2005; MAGGI R., et al.2006). 
Mahdi $\mathrm{H}$ et al. (2015) mostrou que a quimioterapia adjuvante não afetou o risco de recorrência $(25,5 \%$ contra $26.9 \%, p=0,85$ ) na fase I do carcinoma papilífero seroso não invasivo do útero, mesmo em subconjuntos de pacientes submetidos à linfadenectomia ( $20 \%$ versus $23.5 \%, p=0,80)$. No entanto, entre os pacientes que não tiveram linfadenectomia, a quimioterapia adjuvante ou a radiação pélvica foi associada com maior sobrevida livre de progressão $(p=0,04)$ e sobrevida global $(p=0,025)$.

Hirai $\mathrm{M}$ et al. (2002) verificou que a população estudada apresentava invasão linfa-vascular que é uma forma comum de disseminação da doença e obteve redução de recorrência com quimioterapia adjuvante (95\% IC, $-0.51 ;-0.07)$.

A toxidade foi abordada em 2 dos 5 estudos contemplados. Hirai $\mathrm{M}$ et al. (2002) relatou toxidade leve a moderada em 7 pacientes $(30 \%)$ sendo que em $6(26 \%)$ foi necessário interromper o tratamento temporariamente por toxidade hematológica ou infecções. Kuoppala T et al. (2008), durante os 3 cursos de quimioterapia, relatou no grupo de pacientes: náusea, diarreia, alopecia, infecção, anemia e leucopenia. Durante o tratamento o grupo submetido à quimioterapia e radioterapia sofreu mais de anemia e a taxa de sedimentação dos eritrócitos foi maior.

\section{CONSIDERAÇÕES FINAIS}

Através da metanálise não foi possível definir benefício ou malefício com a quimioterapia adjuvante em câncer de endométrio precoce em relação à sobrevivência global e recorrência. Artigos que avaliaram grupos de pacientes mais específicos: como o tipo histológico papilífero seroso ou com invasão linfa-vascular houve benefício no uso de quimioterapia adjuvante quando comparado ao não uso, porém foram avaliadas populações pequenas e os estudos apresentam vieses de seleção. Dessa maneira, são necessários novos estudos com maior número de pacientes, estratificados por tipo histológico e estadio e com critérios definidos de seleção para avaliar a eficácia da quimioterapia adjuvante em câncer de endométrio precoce.

\section{REFERÊNCIAS}

1. AOKI Y, et al. Adjuvant chemotherapy as treatment of high-risk stage I and II endometrial cancer. Gynecol Oncol. 2004 Aug;94(2):333-9.

2. BOER SM, et al. Toxicity and quality of life after adjuvant chemoradiotherapy versus radiotherapy alone for women with high-risk endometrial cancer (PORTEC-3): an open-label, multicentre, randomised, phase 3 trial. The Lancet Oncology.August 2016; Volume 17, No. 8, p1114-1126.

3. BURKE TW, et al. Postoperative adjuvant cisplatin, doxorubicin, and cyclophosphamide (PAC) chemotherapy in women with high-risk endometrial carcinoma. Gynecol Oncol. 1994 Oct;55(1):47-50.

4. BRINTON LA, et al. Etiologic heterogeneity in endometrial cancer: Evidence from a Gynecologic Oncology Group trial. Gynecologic oncology. 2013; 129:277-284.

5. CREASMAN WT, et al. Prognosis of papillary serous, clear cell, and grade 3 stage I carcinoma of the endometrium. Gynecologiconcology. 2004; 95:593-6.

6. CREUTZBERG CL, et al. Outcome of high-risk stage IC, grade 3, compared with stage I endometrial carcinoma patients: The Postoperative Radiation Therapy in Endometrial Carcinoma Trial. JournalofClinicalOncology. 2004, 22.7: 1234-1241.

7. FEHR MK, et al. Chemotherapy of endometrial cancer revisited. Gynakol Geburtshilfliche Rundsch. 1999;39(3):11020.

8. FEHR MK, STREICH MS. The validity of chemotherapy in the treatment of endometrial cancer. Gynakol Geburtshilfliche Rundsch. 2006;46(1-2):34-8.

9. HIRAI M, et al. Adjuvant chemotherapy in stage I uterine endometrial carcinoma. International Journal of Gynaecology and Obstetrics. 2002; 78:37-44.

10. HOWARD DH, et al. A randomized phase III trial in advanced endometrial carcinoma of surgery and volume directed radiation followed by cisplatin and doxorubicin with or without paclitaxel: A Gynecologic Oncology Group study. Gynecologic Oncology, Volume 112, Issue 3, 543 - 552.

11. KELLY MG, et al. Improved survival in surgical stage I patients with uterine papillary serous carcinoma (UPSC) treated with adjuvant platinum-based chemotherapy. Gynecologiconcology. 2005; 8: 353-359.

12. KEYS, HM, et al. A phase III trial of surgery with or without adjunctive external pelvic radiation therapy in intermediate risk endometrial adenocarcinoma: A Gynecologic Oncology Group study. Gynecologiconcology, 2004, 92.3: $744-751$.

REAS | Vol. 11(13) | e1002 | DOI: https://doi.org/10.25248/reas.e1002.2019 Página 8 de 9 
13. KODAMA J, et al. Efficacy and prognostic implications of administering adjuvant chemotherapy to patients with endometrial cancer that is confined to the uterus. European Journalo fObstetrics, Gynecology, and Reproductive Biology. 2007;131(1):76-80.

14. KUOPPALA T, et al. Surgically staged high-risk endometrial cancer: randomized study of adjuvant radiotherapy alone vs. sequential chemo-radiotherapy. Gynecologiconcology. 2008;110(2):190-5

15. LAN C, et al. Should the Optimal Adjuvant Treatment for Patients with Early-Stage Endometrial Cancer with HighIntermediate Risk Factors Depend on Tumor Grade? International Journal of Gynecological Cancer. 2015, 25.8: 1445.

16. MAGGI R, et al. Adjuvant chemotherapy vs radiotherapy in high-risk endometrial carcinoma: results of a randomised trial. British Journal of Cancer. 2006; 95:266-271.

17. MAHDI H et al. Impact of adjuvant chemotherapy and pelvic radiation on pattern of recurrence and outcome in stage I non-invasive uterine papillary serous carcinoma. A multi-institutionstudy. Gynecologiconcology. 2015, 137.2: 239244.

18. MIYAHARA D, et al. Safety and Anti-tumor Effects of Docetaxel Plus Cisplatin in Intermediate- and High-risk Endometrial Cancer. Anticancer Res. 2016 Jul;36(7):3725-9.

19. MOUNTZIOS G, et al. Prognostic factors in patients treated with taxane-based chemotherapy for recurrent or metastatic endometrial cancer: proposal for a new prognostic model. Gynecologic oncology. 2008; 108:130-5.

20. MUNDT AJ, et al. Significant pelvic recurrence in high-risk pathologic stage I--IV endometrial carcinoma patients after adjuvant chemotherapy alone: implications for adjuvant radiation therapy. Int J Radiat Oncol Biol Phys. 2001 Aug $1 ; 50(5): 1145-53$.

21. NAKAMURA T, et al. Evaluation of paclitaxel and carboplatin in patients with endometrial cancer. Gan To Kagaku Ryoho. 2000 Feb;27(2):257-62.

22. NODA K et al. Phase II study of cisplatin in cervical and endometrial carcinomas. Gan To Kagaku Ryoho. 1987 Apr;14(4):1129-35.

23. PUSTILNIK T, BURKE TW. Adjuvant chemotherapy for high-risk endometrial cancer. Semin Radiat Oncol. 2000 Jan;10(1):23-8.

24. RANDALL ME, et al. Randomized phase III trial of whole-abdominal irradiation versus doxorubicin and cisplatin chemotherapy in advanced endometrial carcinoma: A Gynecologic Oncology Group Study. Journal Clinical Oncology. 2006; 24:36-44.

25. ROSE PG. Endometrial carcinoma. New England Journal of Medicine, 1996, 335.9: 640-649.

26. STRINGER CA, et al. Adjuvant chemotherapy with cisplatin, doxorubicin, and cyclophosphamide (PAC) for earlystage high-risk endometrial cancer: a preliminary analysis. Gynecol Oncol. 1990 Sep;38(3):305-8.

27. SUSUMU N, et al. Randomized phase III trial of pelvic radiotherapy versus cisplatin-based combined chemotherapy in patients with intermediate- and high-risk endometrial cancer: a Japanese Gynecologic Oncology Group study. Gynecologic oncology. 2008; 108:226-233.

28. TSUNODA H, et al. Adjuvant chemotherapy with cyclophosphamide, adriamycin, and CDDP (CAP) for high risk endometrial cancer after complete surgery. Nihon Sanka Fujinka Gakkai Zasshi. 1996 Jan;48(1):45-51.

29. VALEJO FAM, TIEZZI DG. Tratamento do Câncer de Endométrio. Femina. Nov 2009 / vol 37 n. 11

30. YAMADA K, et al. Feasibility study of paclitaxel plus carboplatin in patients with endometrial cancer: a Japan Kanto Tumor Board study. J Obstet Gynaecol Res. 2013 Jan;39(1):311-6. 